\title{
Lesión de Dieulafoy de duodeno: hallazgo inusual
}

\author{
An Unusual Finding of a Dieulafoy's Lesion in the Duodenum
}

Angélica Rojas D., MD¹, Germán David Carvajal P., MD², Robin Germán Prieto 0., MD³, Diego Mauricio Aponte M., MD4.

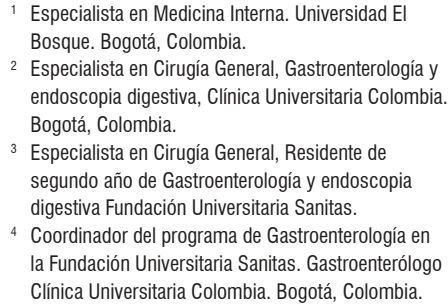

2 Especialista en Cirugía General, Gastroenterología y endoscopia digestiva, Clínica Universitaria Colombia. Bogotá, Colombia.

${ }^{3}$ Especialista en Cirugía General, Residente de segundo año de Gastroenterología y endoscopia digestiva Fundación Universitaria Sanitas.

${ }^{4}$ Coordinador del programa de Gastroenterología en la Fundación Universitaria Sanitas. Gastroenterólogo Clínica Universitaria Colombia. Bogotá, Colombia.

Fecha recibido: $27-11-15$

Fecha aceptado: 25-07-16

\begin{abstract}
Resumen
La lesión de Dieulafoy es una causa poco frecuente de sangrado gastrointestinal alto, pero es una de las causas más frecuentes relacionadas con sangrado oculto y recurrente. La ubicación extragástrica de la lesión de Dieulafoy es rara. Por su localización la lesión de Dieulafoy duodenal es de difícil diagnóstico y manejo. La terapia endoscópica, combinada con inyección de adrenalina más terapia mecánica, reduce el riesgo de resangrado. En este artículo se presenta el caso de un paciente tratado en la Clínica Universitaria Colombia, así como la revisión del tema.
\end{abstract}

\section{Palabras clave}

Sangrado gastrointestinal, lesión de Dieulafoy, duodeno, hemostasia endoscópica.

\section{Abstract}

Dieulafoy's lesions do not usually cause upper gastrointestinal bleeding, but they are one of the most common causes of hidden and recurrent bleeding. An extra-gastric Dieulafoy lesion is rare, and, because of their location, Dieulafoy's lesions in the duodenum are difficult to diagnosis and treat. Endoscopic injection therapy combined with adrenaline injections and mechanical therapy reduce the risk of rebleeding. This article describes the case of a patient treated at the Clínica Universitaria Colombia and reviews the topic of Dieulafoy's lesions.

\section{Keywords}

Gastrointestinal bleeding, Dieulafoy's lesion, duodenal, endoscopic hemostasis.

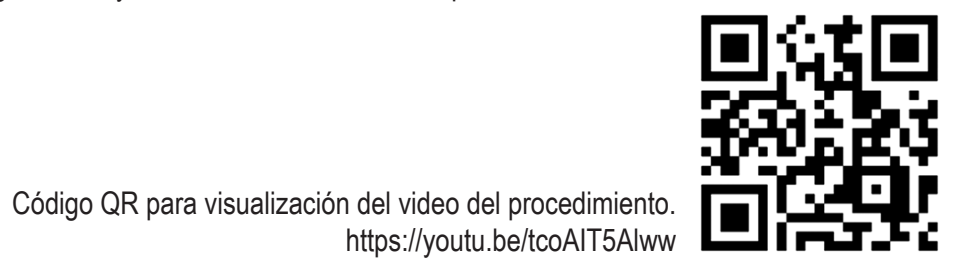

\section{INTRODUCCIÓN}

La incidencia de hemorragia gastrointestinal aguda varía entre 50-150 casos por cada 100000 habitantes por año. En casi el $80 \%$ de los pacientes son causadas por úlceras pépticas y erosiones esofágicas o gastroduodenales (1). El sangrado digestivo oculto representa el $5 \%$ de todas las hemo- rragias gastrointestinales (GI). La lesión de Dieulafoy (LD) es una de las causas de hemorragia digestiva alta oculta, que puede ser potencialmente mortal y representa el $0,1 \%$ de las hemorragias agudas (1).

La mayoría de LD se encuentra en el estómago, dentro de los $6 \mathrm{~cm}$ de la unión gastroesofágica; su localización extragástrica es rara, y en este caso, la ubicación más frecuente 
es la duodenal (2). La falta de conocimiento acerca de la lesión de Dieulafoy aumenta la morbilidad y mortalidad de los pacientes que la padecen. Cabe anotar que se presenta un caso de hemorragia digestiva alta y recurrente, secundaria a lesión de Dieulafoy en el duodeno.

\section{PRESENTACIÓN DEL CASO}

Hombre de 26 años de edad, admitido en el servicio de urgencias por cuadro clínico de 4 días de melenas asociado con hematemesis, astenia, adinamia y un episodio de lipotimia. Niega consumo de antinflamatorios no esteroideos (AINEs) recientemente. Tiene antecedente de hemorragias de vías digestivas recurrentes a la edad de 7, 8, 9 y 15 años; en dichos episodios se le realizó endoscopia de vías digestivas altas (EVDA) sin encontrar foco de sangrado.

En el examen físico inicial se encontró en regular estado general, palidez mucocutánea generalizada, TA: 90/60, FC: $110 \mathrm{lpm}, \mathrm{FR}: 16 \mathrm{rpm}, \mathrm{SaO}_{2}$ : 90\%, abdomen blando, no dolor a la palpación, no signos de irritación peritoneal y tacto rectal positivo para melenas. Hemoglobina de ingreso $15 \mathrm{~g} / \mathrm{dL}$ y hematocrito de $42 \%$. Hemograma de control a las 6 horas con anemización, hemoglobina $13 \mathrm{~g} / \mathrm{dL}$, hematocrito $36 \%$, función renal en rango prerrenal con BUN de 26 y creatinina de 0,84 . Se realiza EVDA y se encuentra normal la mucosa del esófago y del cardias. Estómago: lago mucoso, fundus, cuerpo y antro con presencia de material melénico. Píloro de aspecto normal. Duodeno: entre la primera y segunda porción en la pared posterior, presenta vaso con sangrado activo en jet, compatible con Lesión Dieulafoy (figura 1); se inyecta con solución de adrenalina (1:10.000) y se colocan dos clips hemostáticos, con control total del sangrado (figuras 2 y 3 ).

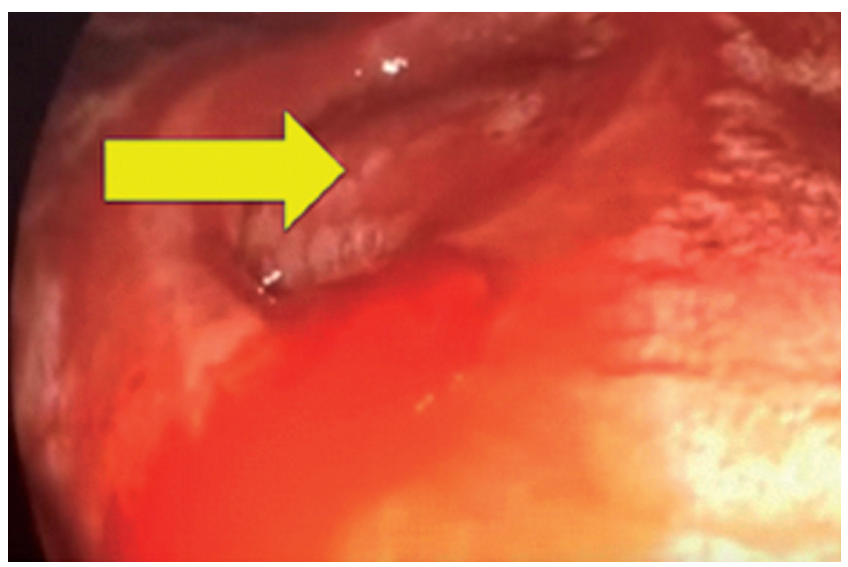

Figura 1. Lesión de Dieulafoy en duodeno pared posterior entre la primera y segunda porción.

Se realiza EVDA de control 12 horas después y no se encuentra sangrado; clips de hemostasia sobre lesión de
Dieulafoy. El paciente evoluciona satisfactoriamente sin inestabilidad hemodinámica y sin resangrado, por lo cual se da egreso y control ambulatorio.

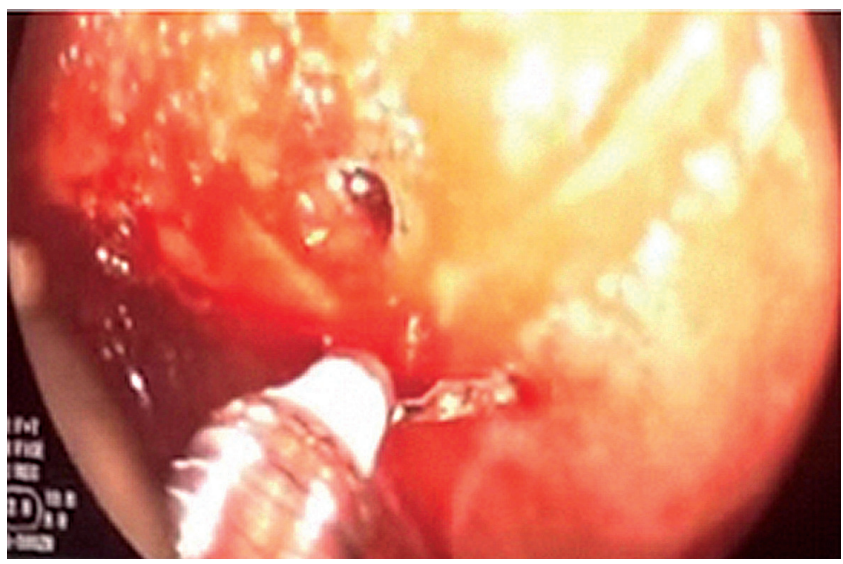

Figura 2. Tratamiento de lesión de Dieulafoy con 2 clips hemostáticos.

\section{DISCUSIÓN}

La lesión de Dieulafoy fue originalmente descrita por Gallard en 1884 como aneurisma miliar del estómago (3); posteriormente, en 1898, fue descrita con más precisión por el cirujano francés Georges Dieulafoy después de realizar un estudio de hemorragia digestiva fatal en tres hombres jóvenes asintomáticos (2). Se caracteriza por una arteria histológicamente normal que tiene un diámetro anormalmente grande $(1-3 \mathrm{~mm})$ y un trayecto tortuoso, que sobresale a través de un pequeño defecto de la mucosa que varía entre 2 y $5 \mathrm{~mm}$. (1). Es importante tener en cuenta que histológicamente la lesión de Dieulafoy es un pequeño defecto de la submucosa con necrosis fibrinoide en su base, que recubre una arteria tortuosa, de paredes gruesas por engrosamiento de la muscularis de la mucosa y fibrosis subintimal, caracterizada por ausencia de inflamación o de úlceras en la mucosa circundante. Normalmente se presenta en adultos y es dos veces más común en hombres que en mujeres. Rara vez se detecta en niños menores de 15 años de edad (3).

Aproximadamente entre el $75 \%$ y el $95 \%$ de estas lesiones se encuentran dentro de los $6 \mathrm{~cm}$ proximales por debajo de la unión gastroesofágica, predominantemente en la curvatura menor. El 5\% restante corresponde a lesiones extragástricas localizadas en el esófago, el duodeno, el yeyuno, el colon y el recto $(4,5)$ (figura 4$)$. Cabe anotar que la patogénesis de la lesión de Dieulafoy es desconocida. En el pasado ha sido considerada como una anormalidad adquirida secundaria al envejecimiento, lo que lleva a la elongación y tortuosidad de una arteria submucosa, aneurismas, aterosclerosis, arteritis o inflamación. Sin embargo, actualmente se cree que la lesión de Dieulafoy puede representar una 


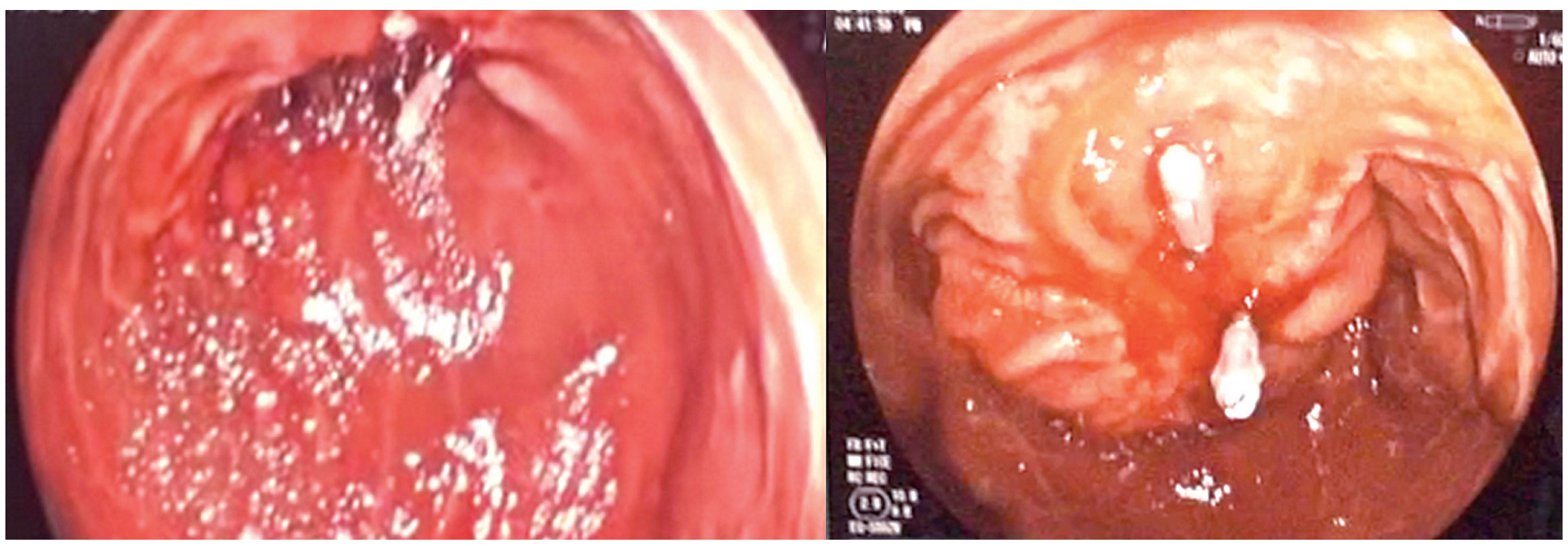

Figura 3. Control del sangrado con inyección de adrenalina más 2 clips hemostáticos en lesión de Dieulafoy duodenal.

malformación vascular congénita $(1,6)$. Estos pacientes se presentan de forma aguda con una hemorragia masiva y en ocasiones recurrente. Los síntomas más frecuentes son hematemesis masiva, melena, hematoquezia, sin dolor abdominal (figura 5).

La endoscopia inicial es eficaz en el diagnóstico de hasta $70 \%$ de los pacientes; sin embargo, se pueden requerir varias endoscopias, incluso aproximadamente el $6 \%$ de los pacientes requieren tres o más para establecer el diagnóstico (1). El no éxito de la endoscopia inicial puede obedecer a la excesiva cantidad de sangre, coágulos y/o lesiones sutiles. Los criterios endoscópicos propuestos para definir la LD son:

- Sangrado arterial activo o micropulsátil desde un defecto mínimo de la mucosa con mucosa circundante normal.

- Visualización de un vaso que sobresale, con o sin sangrado activo, dentro de un defecto mínimo de la mucosa o a través de la mucosa normal circundante.

- Coágulo fresco, adherido con un punto de unión estrecha a un defecto mínimo de mucosa o mucosa de apariencia normal (7).

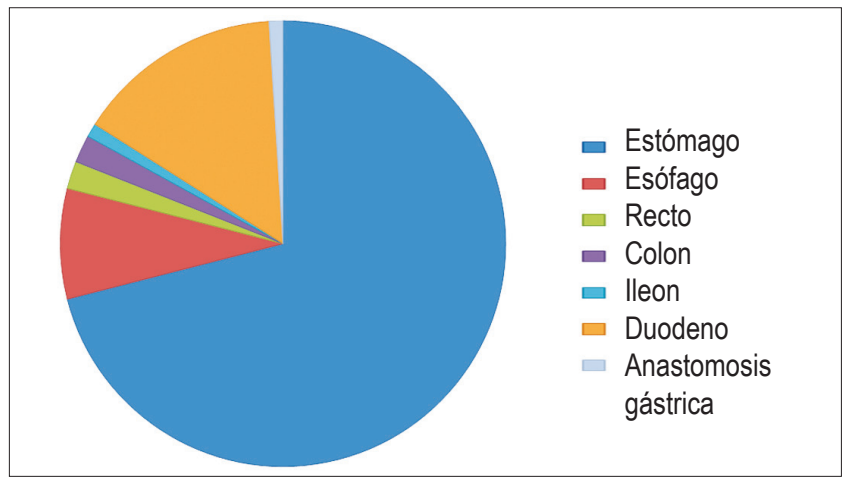

Figura 4. Porcentaje de localización de lesiones de Dieulafoy (1).
Cuando no se localiza la lesión, la enteroscopia permite evaluar el intestino delgado hasta una distancia de $150 \mathrm{~cm}$ desde el píloro, con un rendimiento diagnóstico comprendido entre el $70 \%$ y el $100 \%$ en pacientes con sangrado gastrointestinal oculto (8). También se ha logrado la localización exitosa de la lesión de Dieulafoy con el uso de la cápsula endoscópica, que tiene la ventaja de ser mínimamente invasiva, pero la desventaja de no permitir intervención (1). Hay que considerar que en pacientes con LD el manejo endoscópico se ha convertido en el estándar para el tratamiento, con tasas de éxito hasta del 95\% (9). A propósito, suelen efectuarse diferentes técnicas endoscópicas, la más utilizada es la inyección de solución de adrenalina, ya sea como terapia única o en combinación con otra modalidad (2). Otras técnicas endoscópicas hemostáticas utilizadas son la electrocoagulación, la fotocoagulación láser, la escleroterapia, los hemoclips y la ligadura con bandas.

Con el uso de la electrocoagulación y los métodos de termocoagulación se ha informado el aumento del riesgo de lesión transmural en órganos de paredes delgadas como el

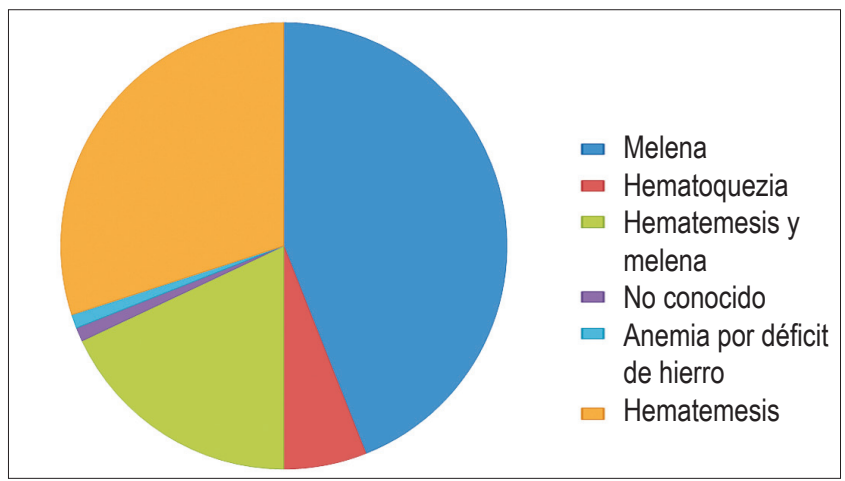

Figura 5. Porcentaje de presentación clínica de la lesión de Dieulafoy (1). 
duodeno, por lo cual se sugiere el uso de hemoclips como un método eficaz y seguro de hemostasia.

Sin embargo, la ligadura con bandas también ha sido informada como una solución eficaz, segura y de bajo costo para el tratamiento de la DL (2).
$\mathrm{Al}$ revisar la literatura para determinar la eficacia de los diferentes métodos de tratamiento endoscópicos, Chung y colaboradores (10) compararon los resultados de la inyección de solución salina hipertónica frente a la terapia mecánica con hemoclips o ligadura con banda (24 pacientes). La

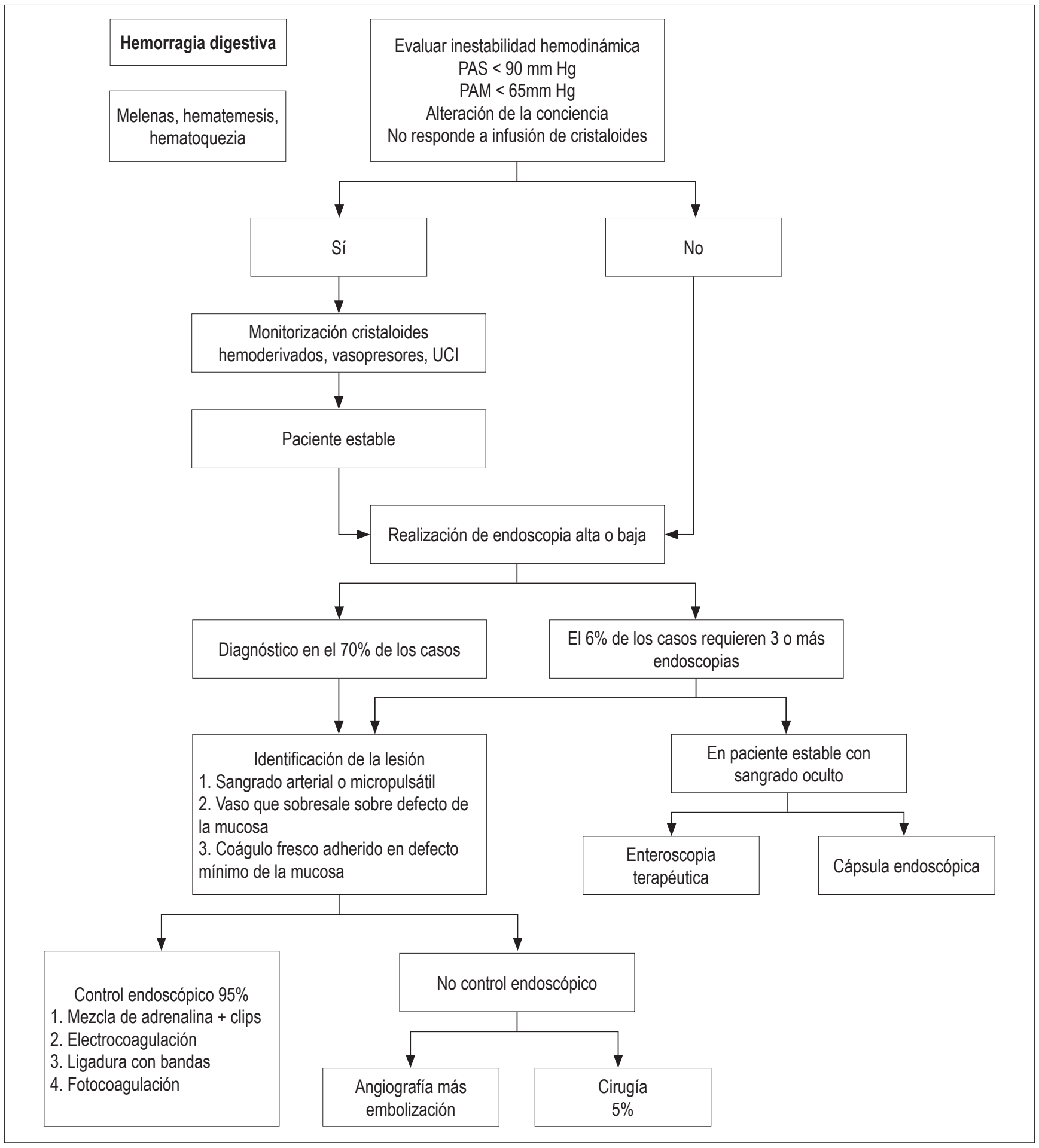

Figura 6. Algoritmo de tratamiento de la lesión de Dieulafoy 
tasa de control inicial del sangrado fue mayor en el grupo de pacientes tratados con la terapia mecánica, mientras que la tasa de resangrado fue significativamente mayor en el grupo tratado con la solución salina hipertónica.

En un ensayo clínico realizado por Park y colaboradores (11) se demostró el uso de los hemoclips como una terapia significativamente más eficaz en la prevención de las hemorragias recurrentes. Por otro lado, Karaahmet y colaboradores (12) sugieren que la combinación endoscópica de la inyección de adrenalina y las terapias mecánicas son superiores a la monoterapia en el tratamiento de DL. La angiografía y la embolización se indican para pacientes con sangrado activo que no son susceptibles de tratamiento endoscópico y/o quirúrgico, o con sangrado oculto e inaccesible (13).

Finalmente, la resección quirúrgica está reservada para el $5 \%$ de los casos que son refractarios al manejo endoscópico o a los métodos angiográficos. En la figura 6 mostramos el algoritmo para el estudio y para el tratamiento de la lesión de Dieulafoy.

\section{CONCLUSIÓN}

La lesión duodenal de Dieulafoy es una patología poco frecuente, causante de hemorragia grave y recurrente de las vías digestivas altas. Un adecuado diagnóstico endoscópico temprano es fundamental. Actualmente se dispone de múltiples técnicas endoscópicas eficaces para el control del sangrado; sin embargo, la terapia combinada de inyección de adrenalina, complementada con terapia mecánica, reduce el riesgo de resangrado.

\section{REFERENCIAS}

1. Baxter M. Dieulafoy's lesion: current trends in diagnosis and management. Ann R Coll Surg Engl. 2010;92(7):548-554
2. Mohammed Ibrarullah and Gajanan D Wagholikar. Dieulafoy's lesion of duodenum: a case report. BMC Gastroenterology. 2003,31(3):2.

3. Chaer R, Helton WS. Dieulafoy's disease. Am Coll Surg. 2003;196:290-6.

4. McClave SA, Goldschmid S, Cunningham JT and Boyd WP Jr. Dieulafoy's cirsoid aneurysm of the duodenum. Dig Dis Sci. 1988,33(7):801-805.

5. Norton ID, Petersen BT, Sorbi D, Balm RK, Alexander GL and Gostout CJ. Management and long-term prognosis of Dieulafoy lesion. Gastrointest Endosc. 1999, 50(6):762-767

6. Dincer Avlan, Ali Nayci, Engin Altintas. An unusual cause for massive upper gastrointestinal bleeding in children: Dieulafoy's lesion. Pediatr Surg Int. 2005;21(5):417-418.

7. Dy NM, Gostout CJ and Balm RK. Bleeding from the endoscopically-identified Dieulafoy lesion of the proximal small intestine and colon. Am J Gastroenterol. 1995;90(1):108-111.

8. Al-Mishlab T, Amin AM and Ellul JM. Dieulafoy's lesion: an obscure cause of GI bleeding. J R Coll Surg Edinb. 1999;44(4):222-225.

9. Sone Y, Kumada T, Toyoda H, et al. Endoscopic management and follow up of Dieulafoy lesion in the upper gastrointestinal tract. Endoscopy. 2005;37(5):449-53.

10. Chung IK, Kim EJ, Lee MS, et al. Bleeding Dieulafoy's lesions and the choice of endoscopic method: comparing he hemostatic efficacy of mechanical and injection methods. Gastrointest Endosc. 2000;52(6):721-4.

11. Park CH, Joo YE, Kim HS, Choi SK, Rew JS, Kim SJ. A prospective, randomized trial of endoscopic band ligation versus endoscopic hemoclip placement for bleeding gastric Dieulafoy's lesions. Endoscopy. 2004;36(8):677-81.

12. Karaahmet Fatih, Kilıncalp S, Coskun. The efficiency of endoclips in maintaining the gastrointestinal bleeding-related Dieulafoy's lesion. 2015;9.

13. Alomari A, Fox V, Kamin D. Embolization of a bleeding Dieulafoy lesion of the duodenum in a child. Case report and review of the Literature. Journal of Pediatric Surgery. 2013; 48(1):e39-41. 